\title{
Feasibility of a Tertiary Hospital Antimicrobial Stewardship Ward Round Using an Electronic Prescribing System-A Pilot Study
}

\author{
Niall L. Hamilton ${ }^{1}$ Sharon J. Gardiner ${ }^{1,2,3}$ Qian Yi Chuah ${ }^{1}$ Paul K.L. Chin ${ }^{1,4}$ Simon C. Dalton ${ }^{2}$ \\ Sarah C. L. Metcalf ${ }^{2}$
}

${ }^{1}$ Department of Clinical Pharmacology, Canterbury District Health
Board, Christchurch, New Zealand
${ }^{2}$ Department of Infectious Diseases, Canterbury District Health
Board, Christchurch, New Zealand
${ }^{3}$ Pharmacy Services, Canterbury District Health Board, Christchurch,
New Zealand
${ }^{4}$ Department of Medicine, University of Otago, Christchurch, New Zealand

Address for correspondence Sharon J. Gardiner, BPharm(Hons), MClinPharm, PhD, Department of Infectious Diseases, Christchurch Hospital, PO Box 4710, Christchurch 8140, New Zealand (e-mail: sharon.gardiner@cdhb.health.nz).

ACI Open 2020;4:119-125.

\begin{abstract}
Keywords

- electronic prescriptions

- pharmacy

- medicine

- antimicrobial stewardship

- antimicrobial resistance

- Infectious Diseases Service

- resident doctor

Objectives The aim was to conduct a pilot study to determine staff resource requirements for an antimicrobial stewardship (AMS) ward round informed by electronic prescriptions, and the number of restricted antimicrobial prescriptions that would prompt a ward round recommendation.

Methods Prescription data on 26 restricted antimicrobial agents (which have specific prescribing criteria defined by the national drug funding agency) were extracted from the electronic prescribing and administration system (MedChart). A language query was used for specific antimicrobial names on Mondays, Wednesdays, and Fridays over 4 weeks. Prescriptions that had ceased or had an appropriate indication documented were excluded. The remaining prescriptions were evaluated in the ward round upon clinical record review with theoretical AMS recommendations made and time requirements recorded. The ward rounds were performed by two clinical staff, a doctor and pharmacist.

Results In 12 days, 622 prescriptions were extracted. Of these, 66 were evaluated in ward rounds, with $67 \%(44 / 66)$ being for ciprofloxacin or piperacillin-tazobactam. Theoretical AMS recommendations were made in $61 \%$ (40/66) of cases, $45 \%(18 / 40)$ being to use a narrower spectrum agent, $30 \%$ (12/40) to consult the Infectious Diseases Service, and 23\% (9/40) to stop antimicrobial therapy. Data extraction took an analyst approximately 15 minutes, screening by a doctor approximately 20 minutes, and ward rounds approximately 41 minutes per day.

Conclusion Our approach required, in total, approximately 100 clinical staff minutes per day to screen approximately 50 prescriptions and identify and evaluate approximately four prescriptions and showed clinical value. Resource planning should also consider Infectious Diseases and/or Microbiology (physician and service) involvement, and audit capability.
\end{abstract}

received

November 3, 2019

accepted

July 30, 2020
DOI https://doi.org/

10.1055/s-0040-1716749.

ISSN 2566-9346. (c) 2020 Georg Thieme Verlag KG

Stuttgart · New York
License terms

(c) (i) 


\section{Background and Significance}

The emergence and spread of resistant microorganisms is a "global crisis" that is "one of the greatest threats to health."1 It is attributed, in part, to selection pressures from widespread use of antimicrobial agents. ${ }^{2}$ The World Health Organization has released a plan for combating antimicrobial resistance (AMR) as have over 70 countries including the United States of America and New Zealand. ${ }^{3-5}$ The strategies include antimicrobial stewardship (AMS) as a core objective.

AMS is an umbrella term encompassing coordinated strategies to promote appropriate antimicrobial prescribing and improve patient outcomes including reducing AMR. Key international organizations, including the Infectious Diseases Society of America with the Society for Healthcare Epidemiology of America, have released recommendations for the structure and implementation of AMS programs within health care institutions. ${ }^{6}$ Two major approaches recommended for optimizing antimicrobial prescribing in the hospital setting are formulary restriction with preauthorization for use of certain antimicrobial agents (a restrictive "front-end" strategy), and prospective audit with intervention and feedback (a persuasive "back-end" strategy). Both methods improve outcomes related to antimicrobial use including the appropriateness of prescribing, and reduce occurrence of infections caused by Clostridium difficile and multidrug-resistant bacteria. ${ }^{6,7}$

Canterbury District Health Board (DHB) provides publically funded health care for approximately 560,000 people living in Canterbury, New Zealand. The largest Canterbury DHB facility is Christchurch Hospital, which includes the geographically co-located Christchurch Women's Hospital ("Christchurch Hospital campus"). This tertiary referral facility comprises approximately 800 beds and has a full range of medical, surgical, maternity, and pediatric services including intensive care, and bone marrow and renal transplant units.

The national drug buying agency, PHARMAC, $^{8}$ provides a restrictive component to hospital prescribing with antimicrobial agents, in broad terms, able to be prescribed without restriction (e.g., amoxicillin), or only for certain indications (e.g., clarithromycin) and/or by certain specialties (e.g., ciprofloxacin use requires infectious diseases physician or clinical microbiologist support). To extend the AMS program at Canterbury DHB, the AMS committee plans to establish an effective and sustainable prospective audit and feedback service in the form of an "AMS ward round." This post-prescription strategy involves a specialist multidisciplinary AMS team reviewing antimicrobial therapy for individual patients with the view to making recommendations to promote optimal antimicrobial use, such as to change from an intravenous to oral agent or de-escalate to a narrower spectrum agent. AMS ward rounds can improve antimicrobial therapy for both current and future patients by providing educational opportunities, and are more readily accepted by clinicians than a restrictive approach. ${ }^{6}$

\section{Objectives}

The aim of this pilot study was to determine the feasibility and ongoing resource requirements for AMS ward rounds at Canterbury DHB hospitals using the existing electronic prescribing and administration (ePA) system (MedChart) as the primary strategy for identifying patients for review. Specifically, we sought to ascertain the:

- Number of prescriptions that would require review of the physical notes.

- Time taken to perform the data extraction and interrogation, and subsequent review of the notes.

- Suggested recommendation following review, and

- Number of prescriptions that prompted a recommendation of a formal infectious disease's referral and review.

\section{Methods}

The inclusion criteria for this feasibility study were adult inpatients $\geq 18$ years of age admitted under a medical or surgical service at the Christchurch Hospital campus who were electronically prescribed one or more of 26 selected restricted antimicrobial agents ( - Fig. 1). Excluded patients were those in the Emergency Department or Intensive Care Unit (e-PA not implemented in these services), or under the care of the Respiratory (several restricted antimicrobials can be used by respiratory physicians), Hematology (existing close involvement with the Infectious Diseases and Clinical Microbiology Services), or Pediatric and Neonatal (outside AMS committee scope) services.

-Fig. 1 outlines the process for identifying patients for inclusion in the AMS ward rounds. For each day that the AMS ward round was undertaken, a health information analyst used locally designed structured query language to extract all active restricted antimicrobial prescriptions for all adult inpatient areas (other than the Emergency Department and Intensive Care Unit) from the independent and nonintegrated e-PA software used at Canterbury DHB (MedChart, version 8.1.1, DXC Technology, Tysons, Virginia, United States). At our institution, restricted antimicrobial prescriptions do not require prior authorization for use; instead, a persuasive approach post-prescription is used to encourage appropriate antimicrobial use post-prescribing. A direct, configurable, data report was not available as part of the MedChart software. The data source for the extracted prescriptions was the Canterbury DHB back-up file of the e-PA system that is conducted daily at 0200 hours (same day as the subsequent AMS review). Real time prescription extraction was not feasible due to resource constraints and the possibility that direct extraction from the live system could interfere with it and thus disrupt patient care. Extracted data included the following for each restricted antimicrobial prescription: associated National Heath Index number (nationally unique patient identifier for health and disability services), and the medicine's name, dose, frequency, route of administration, and start time. The analyst exported these data into an analysis program (Tableau Desktop 8.1, Tableau, Seattle, Washington, United States) to further filter and organize 


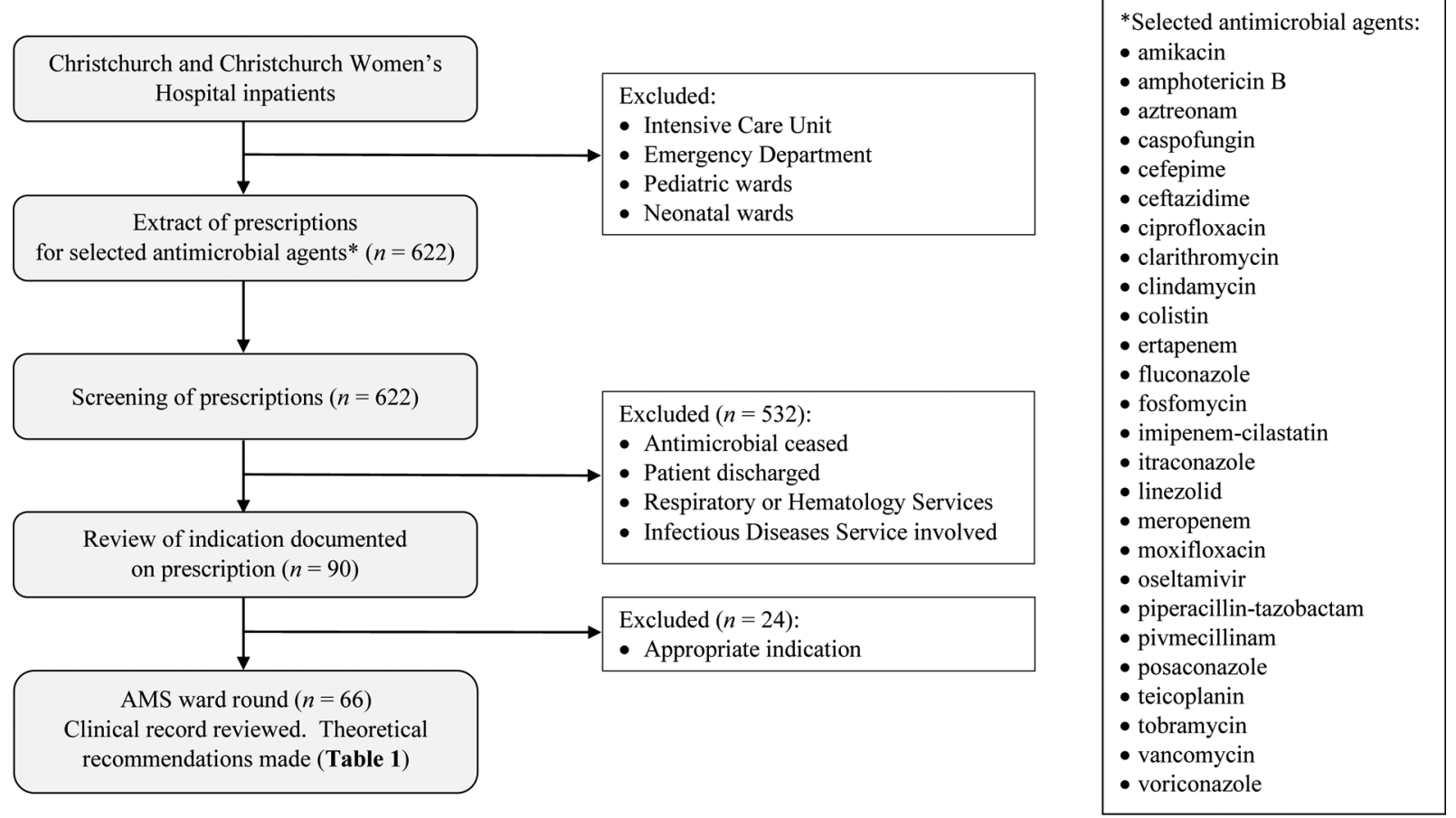

Fig. 1 Process for identifying inpatients for inclusion in AMS ward rounds. AMS, antimicrobial stewardship.

the prescriptions by patient and to provide data visualization before exporting into a spreadsheet (Microsoft Excel 2013). A registered medical officer (resident doctor) undertaking specialist training in both clinical pharmacology and infectious diseases then reviewed the extracted prescriptions against the active electronic clinical record manually to determine if the identified prescription was still current, if the patient was still an inpatient and in a relevant ward or clinical area, and if an appropriate indication (compliant with restrictions) was part of the prescription. Prescribers at our institution are not required to include indications on antimicrobial prescriptions. So, lack of an "appropriate" indication in the prescription does not necessarily mean use is inappropriate. The extracted prescriptions and associated patient list were also compared against the electronic database of patients currently under review by the Infectious Diseases Service to avoid work duplication. The remaining patients and their antimicrobial prescriptions were included in the AMS ward round. The data extraction and analysis was conducted securely and stored on the hospital computer servers, which requires staff to login with a password.

None of the patients were physically reviewed on the ward round. Instead, paper and electronic records were reviewed for each included patient. The ward round was performed by two multidisciplinary staff members, comprising a senior resident doctor and an AMS pharmacist as recommended by key international guidelines. ${ }^{9,10}$ The pilot ward round was developed by the Infectious Diseases Service in conjunction with both the Pharmacy and the Clinical Pharmacology Departments. Particular attention was paid to compliance with Canterbury DHB guidelines, optimal dosing (e.g., against renal function, antimicrobial plasma concentrations), and microbiology results. A theoretical recommendation was made from a set list ( $>$ Table 1 ) by both reviewers and a consensus decision reached; if agreement could not be reached, it was discussed with an infectious diseases physician who determined the final recommendation. If more than one recommendation was possible (e.g., change of route plus de-escalation), only the highest priority recommendation (i.e., de-escalation, in this case) was recorded. The recommendation for each prescription was recorded in the study database but not documented in the patients' clinical records or communicated to the primary team. This was because this was a feasibility study and implementation of AMS ward rounds would require prior engagement with executive management and clinical services to promote "buy-in." However, if the antimicrobial regimen was perceived to potentially be ineffective due to the spectrum of activity or planned duration of treatment, the primary team was advised to contact the Infectious Diseases Service for advice. The limitation of time and personnel for patient reviews meant that the diagnosis from the attending team was considered valid. In cases of diagnostic uncertainty impacting on the antimicrobial choice or duration, we recommended formal referral and review by the Infectious Diseases Service.

Extraction and review of prescriptions were undertaken on 12 days, as 3 days each week (Monday, Wednesday, and Friday) across 4 nonconsecutive weeks (to coincide with the availability of the two clinical staff members) between August 14, 2017 and September 29, 2017. The limit of 3 days per week was dictated by available personnel resource and was spaced out to attempt to provide coverage of prescriptions across the whole week. The time taken for each component (extraction, screening, and the ward rounds) was recorded each day. 
Table 1 Potential recommendations following review of a restricted antimicrobial agent in the AMS ward rounds

\begin{tabular}{|l|l|}
\hline Recommendation & Rationale for decision \\
\hline 1. No intervention needed & Indication and dosing regimen are inappropriate. \\
\hline 2. Stop antimicrobial & Spectrum overlaps with another antimicrobial or antimicrobial not indicated. \\
\hline 3. Start antimicrobial & Inadequate antimicrobial cover for indication. \\
\hline 4. Change dose & Dose inappropriate for weight, renal function, or antimicrobial concentration. \\
\hline 5. Change duration of treatment & Specified duration too long or short. \\
\hline 6. Change frequency of administration & Incorrect frequency selected or adjustment for renal function required. \\
\hline 7. Change route of administration & Antimicrobial suitable for IV to oral switch, or route inappropriate for formulation. \\
\hline $\begin{array}{l}\text { 8. De-escalation of empiric broad } \\
\text { spectrum to narrow spectrum } \\
\text { antimicrobial }\end{array}$ & Microbial culture results or expected microflora for condition support change. \\
\hline $\begin{array}{l}\text { 9. Escalation of narrow spectrum to } \\
\text { broader spectrum antimicrobials }\end{array}$ & $\begin{array}{l}\text { Patient has deteriorated clinically, or culture results or expected } \\
\text { microflora for condition support change. }\end{array}$ \\
\hline $\begin{array}{l}\text { 10. Refer to infectious diseases service } \\
\text { Patient requires physical review, further investigations, assessment, } \\
\text { and/or ongoing follow-up by the Infectious Diseases Service } \\
\text { (beyond scope of AMS ward round). }\end{array}$ \\
\hline
\end{tabular}

Abbreviations: AMS, antimicrobial stewardship; IV, intravenous.

Table 2 Review process for the initial 622 prescriptions extracted from CDHB e-prescription and administration software

\begin{tabular}{|c|c|c|c|c|c|c|}
\hline \multirow[t]{2}{*}{$\begin{array}{l}\text { Restricted } \\
\text { antimicrobial }^{\mathrm{a}}\end{array}$} & \multirow{2}{*}{$\begin{array}{l}\text { Number of } \\
\text { prescriptions } \\
\text { extracted }\end{array}$} & \multicolumn{5}{|c|}{$\begin{array}{l}\text { Number of prescriptions (\% of total number of prescriptions extracted for each } \\
\text { antimicrobial) }\end{array}$} \\
\hline & & $\begin{array}{l}\text { Ceased at the } \\
\text { time of } \\
\text { prescription } \\
\text { review }\end{array}$ & $\begin{array}{l}\text { Prescribed for } \\
\text { Hematology or } \\
\text { Respiratory } \\
\text { Service Patient }\end{array}$ & $\begin{array}{l}\text { Prescribed for } \\
\text { patient known } \\
\text { to the Infectious } \\
\text { Diseases Service }\end{array}$ & $\begin{array}{l}\text { Appropriate } \\
\text { indication } \\
\text { documented } \\
\text { on the } \\
\text { prescription }\end{array}$ & $\begin{array}{l}\text { Reviewed } \\
\text { on AMS } \\
\text { ward round }\end{array}$ \\
\hline Piperacillin-tazobactam & 222 & $63(28 \%)$ & $105(47 \%)$ & $26(11 \%)$ & $8(4 \%)$ & $20(9 \%)$ \\
\hline Ciprofloxacin & 137 & $82(60 \%)$ & $19(14 \%)$ & $6(4 \%)$ & $6(4 \%)$ & $24(17 \%)$ \\
\hline Meropenem & 62 & 20 (32\%) & $22(35 \%)$ & 12 (19\%) & $1(8 \%)$ & $7(11 \%)$ \\
\hline Vancomycin & 59 & $23(39 \%)$ & $13(22 \%)$ & $12(20 \%)$ & $6(10 \%)$ & $5(8 \%)$ \\
\hline Clindamycin & 40 & $24(60 \%)$ & $6(15 \%)$ & $4(10 \%)$ & 0 & $6(15 \%)$ \\
\hline Ceftazidime & 21 & $4(19 \%)$ & $17(81 \%)$ & 0 & 0 & 0 \\
\hline Clarithromycin & 17 & $11(65 \%)$ & $1(6 \%)$ & $3(18 \%)$ & $2(11 \%)$ & 0 \\
\hline Tobramycin & 17 & $7(41 \%)$ & $9(52 \%)$ & 0 & 0 & $1(6 \%)$ \\
\hline Fluconazole & 16 & $7(43 \%)$ & $5(31 \%)$ & $1(6 \%)$ & $1(6 \%)$ & $2(12 \%)$ \\
\hline Posaconazole & 8 & 0 & $8(100 \%)$ & 0 & 0 & 0 \\
\hline Ertapenem & 7 & $4(57 \%)$ & 0 & $3(43 \%)$ & 0 & 0 \\
\hline Imipenem-cilastatin & 4 & $1(25 \%)$ & $3(75 \%)$ & 0 & 0 & 0 \\
\hline Teicoplanin & 4 & $1(25 \%)$ & 0 & $3(75 \%)$ & 0 & 0 \\
\hline Cefepime & 3 & $1(33 \%)$ & $2(67 \%)$ & 0 & 0 & 0 \\
\hline Linezolid & 2 & $1(50 \%)$ & 0 & 0 & 0 & $1(50 \%)$ \\
\hline Valganciclovir & 2 & 0 & $2(100 \%)$ & 0 & 0 & 0 \\
\hline Amphotericin B & 1 & 0 & $1(100 \%)$ & 0 & 0 & 0 \\
\hline Total & 622 & $249(40 \%)$ & $213(34 \%)$ & $70(11 \%)$ & $24(4 \%)$ & $66(11 \%)$ \\
\hline
\end{tabular}

Abbreviations: AMS, antimicrobial stewardship; CDHB, Canterbury District Health Board.

${ }^{a}$ Nine restricted antimicrobials were not prescribed during the feasibility project: amikacin, aztreonam, caspofungin, colistin, fosfomycin, itraconazole, moxifloxacin, pivmecillinam, and oseltamivir. 


\section{Results}

\section{Initial Data Extract $(n=622)$}

A total of 622 prescriptions for restricted antimicrobials were extracted from MedChart, comprising a median (range) of 51 (16-85) prescriptions per ward round day. The vast majority (84\%) of the extracted prescriptions (520/622) were for five antibacterial agents: piperacillin-tazobactam $(n=222)$, ciprofloxacin $(n=137)$, meropenem $(n=62)$, vancomycin $(n=59)$, and clindamycin $(n=40)$ (-Table 2).

\section{Prescriptions Excluded from the AMS Ward Rounds $(n=556)$}

-Fig. 1 and -Table 2 show the number of excluded prescriptions at each step of the screening process, from data extraction until inclusion in the ward rounds. Of the 622 prescriptions extracted, 532 (86\%) were excluded at the screening stage: $47 \%$ (249/532) because the prescription was stopped or the patient was discharged between 0200 hours (time that the MedChart back-up file, used as the source for the extract, was performed each day) and 0800 to 1000 hours (when the screening process took place); 40\% (213/532) because the patient was under the care of the Respiratory or Hematology Services; 13\% (70/532) because the patient was known to the Infectious Diseases Service. A further 24 prescriptions were excluded because there was a guideline-compliant indication and dosing regimen entered in MedChart.

\section{Prescriptions Included in the AMS Ward Rounds ( $n=66)$}

Eleven percent of extracted prescriptions (66/622) were included in the AMS ward rounds. There was a median (range) of four prescriptions (0-15) per ward round day. Upon review of patient clinical records, existing antimicrobial therapy was assessed as appropriate in $39 \%$ of cases (26/ $66)$ with no further action required. Theoretical recommen- dations were made in the remaining $61 \%$ of cases (40/66) (-Table 3): 45\% (18/40) were to de-escalate to a narrower spectrum agent, $30 \%(12 / 40)$ were a referral to the Infectious Diseases Service because of a complex clinical picture, 23\% (9/40) were to cease antimicrobial therapy, and 3\% (1/40) were to change administration route. Two of the 66 prescriptions required discussion with an infectious diseases physician to determine the recommendation. Two-thirds (66\%) of the prescriptions were for ciprofloxacin (24/66) or piperacillin-tazobactam (20/66) (-Table 3).

Direct advice (actual rather than theoretical recommendations) to consult with the Infectious Diseases Service was given to clinical teams in two cases when the current antimicrobial prescription was considered inadequate for treating the infection-one case involved the prescribed agent not having activity against the pathogen isolated, and a second case involved the prescribed antimicrobial regimen being of inadequate duration for bacteremia.

\section{Resource Requirements for the AMS Ward Rounds}

The set-up and day-to-day running of our AMS ward rounds required three people (a health information analyst, a resident doctor, and an AMS pharmacist) plus oversight from infectious diseases physicians. The health information analyst required approximately 15 minutes prior to each ward round to filter the extracted e-prescription data and export it into a spreadsheet. Pre-ward round screening of the extracted prescriptions by a doctor or pharmacist took a median (range) of 20 (20-50) minutes per day. The ward round itself took a doctor and pharmacist a median (range) of 28 (10-90) minutes per day for the clinical aspects (reviewing paper and electronic records, and recommendation decisions), plus a further 13 (1-30) minutes for nonclinical aspects (travel time, locating physical notes, and computers on the ward). Additional discussion of two cases with an infectious diseases physician took around 5 minutes each.

Table 3 Recommendations from the AMS ward round review of restricted antimicrobials

\begin{tabular}{|c|c|c|c|c|c|c|}
\hline \multirow{2}{*}{$\begin{array}{l}\text { Restricted } \\
\text { antimicrobial }^{\mathrm{a}}\end{array}$} & \multirow{2}{*}{$\begin{array}{l}\text { Number of } \\
\text { prescriptions } \\
\text { reviewed on } \\
\text { AMS ward round }\end{array}$} & \multirow{2}{*}{$\begin{array}{l}\text { Prescriptions } \\
\text { with a } \\
\text { theoretical AMS } \\
\text { recommendation }\end{array}$} & \multicolumn{4}{|c|}{ Recommendation (number of prescriptions) $^{a}$} \\
\hline & & & $\begin{array}{l}\text { Change } \\
\text { route }\end{array}$ & $\begin{array}{l}\text { Stop } \\
\text { antimicrobial }\end{array}$ & $\begin{array}{l}\text { De-escalate } \\
\text { to narrower } \\
\text { spectrum }\end{array}$ & $\begin{array}{l}\text { Refer to } \\
\text { Infectious } \\
\text { Diseases Service }\end{array}$ \\
\hline Ciprofloxacin & 24 & $20(83 \%)$ & 0 & 5 & 10 & 5 \\
\hline Piperacillin-tazobactam & 20 & $10(50 \%)$ & 0 & 0 & 8 & 2 \\
\hline Meropenem & 7 & $4(57 \%)$ & 0 & 1 & 0 & 3 \\
\hline Clindamycin & 6 & $3(50 \%)$ & 1 & 2 & 0 & 0 \\
\hline Vancomycin & 5 & $2(40 \%)$ & 0 & 1 & 0 & 1 \\
\hline Fluconazole & 2 & $1(50 \%)$ & 0 & 0 & 0 & 1 \\
\hline Tobramycin & 1 & 0 & 0 & 0 & 0 & 0 \\
\hline Linezolid & 1 & 0 & 0 & 0 & 0 & 0 \\
\hline Total & 66 & $40(61 \%)$ & 1 & 9 & 18 & 12 \\
\hline
\end{tabular}

Abbreviation: AMS, antimicrobial stewardship.

${ }^{\text {a } T h e r e ~ w e r e ~ n o ~ t h e o r e t i c a l ~ A M S ~ r e c o m m e n d a t i o n s ~ f o r ~ c h a n g e ~ i n ~ d o s e, ~ s t a r t ~ a n t i m i c r o b i a l, ~ c h a n g e ~ d u r a t i o n, ~ c h a n g e ~ f r e q u e n c y, ~ o r ~ e s c a l a t i o n ~ f r o m ~}$ narrower to broader spectrum agent. 


\section{Discussion}

We aimed to determine the feasibility and resource requirements for an AMS ward round at our approximately 800 bed tertiary hospital campus using the existing e-PA system (MedChart) to avoid additional software costs. We showed that MedChart, which is used in various health care facilities in New Zealand, Australia, and the United Kingdom, can be an effective tool for patient identification. However, there were multiple personnel skillsets needed, unconsidered resourcing components, and several sizeable inefficiencies for the work-up required for each ward round:

1. Human resourcing: Three core staff were needed. A technical expert established the initial process for data extraction and performed extractions for each ward round (approximately 15 minutes each), which may become automated in time. The clinical skillset (doctor and pharmacist) for the ward rounds required a median daily time commitment of $\sim 100$ minutes (screening by one person, ward round by two people), and followed the recommended multidisciplinary approach for AMS ward rounds. ${ }^{9,10}$ Future planning should include resourcing for infectious diseases physicians and/or clinical microbiologists, as prescribing practices are more likely to be influenced with active and visible senior clinician involvement. ${ }^{11}$ Estimated median time for senior clinician involvement in the ward round proper is around 40 minutes per day (approximately 28 minutes for clinical aspects, approximately 13 minutes for nonclinical aspects). Further, 30\% of our AMS recommendations were to consult the Infectious Diseases Service, and so the impact of the rounds on this service must also be considered.

2. High attrition rate: Clinical screening of the extracted prescriptions to manually identify those for review in the ward rounds had a very high attrition rate of approximately $90 \%$. From a total of 622 (approximately 50 per ward round day) prescriptions extracted, only 66 (approximately 4 per ward round day) were included. The greatest loss (40\%) was of prescriptions (249/622) ceased between 0200 hours (time the Canterbury DHB back-up file was created) and 0800 to 1000 hours when screening occurred. This is not surprising as screening was done on only 3 days per week (Mondays, Wednesdays, and Fridays). Thus, there was a variable lag of 8 to 80 hours between creation of the source document, and prescription extraction, and screening (e.g., "current" prescriptions at 0200 hours on Saturday, Sunday, and Monday mornings were combined for screening during working hours on Monday morning). A data source that approaches "real time" would improve screening efficiency, while more frequent ward rounds (e.g., 5 working days) would increase the volume of prescriptions reviewed. Realtime data extraction was not attempted as this would involve directly extracting data from the live MedChart, and therefore potentially interfere with active MedChart records and disrupt patient care. This may change with future versions of MedChart, and would require the associated increased availability of expert personnel to process the data. Further, we could not identify any improvements that could be made to our data interrogation query that would reduce the screening burden to make better use of the AMS team's time.

3. Excluded services: We chose to exclude three adult services with high volumes of antimicrobial prescribing and more complex patients. Our Hematology and Intensive Care Units already have meetings two to three times a week with both the Infectious Diseases and Clinical Microbiology Services to discuss patients on antimicrobial therapy, including a stewardship focus. The Respiratory Service was excluded as several of the restricted antimicrobials (e.g., ceftazidime and piperacillin-tazobactam) can be prescribed with endorsement of a respiratory physician, but we recognize that our path forward for AMS ward rounds should include active engagement with this service.

The volume and nature of the theoretical recommendations made in our pilot study indicate that an AMS ward round service would be beneficial at our institution, although it would be important to evaluate how many recommendations were adopted and the associated patient outcomes. More than half (61\%) of the reviewed antimicrobial prescriptions (40/66) could be improved from an AMS perspective, largely via de-escalation to a narrower spectrum agent (45\%), consultation with Infectious Diseases (30\%), or cessation of antimicrobial therapy (23\%).

Internationally, AMS wards rounds have been shown to produce clinical benefits, and to reduce cost and adverse events. ${ }^{12}$ A range of methods is used for patient/prescription identification, including reviewing all drug charts in specific clinical areas, referral from clinical staff, and use of "high interest" antimicrobial agents. ePA systems can offer a useful starting place to identify patients for prescription review. Software packages, such as the ICNet pharmacy module ("ABX Steward"; Baxter Healthcare Corporation, Deerfield, Illinois, United States), should be evaluated as an alternative model that could link into MedChart to extract prescribing data and avoid the need for the work-around steps we have needed. This has the ability to link together prescribing data and laboratory data to identify patients in "real time" who might benefit from review. Use of this ICNet module has been demonstrated to facilitate case finding, increase productivity and audit capacity of the AMS team, and help optimize infection management. ${ }^{13}$ ICNet is capable of using MedChart as its source of prescription data, and this could be explored at our facility in the future.

Our study had several limitations, some of which have already been discussed above. The e-PA system used in this study was MedChart, which potentially limits the applicability of our results to other e-PA systems. However, this is a novel aspect of our study as we are not aware of any other published AMS studies that have used MedChart in isolation. Further, our approach to identifying patients for review, especially the comprehensive identification of "restricted" prescriptions, is not replicable for paper prescribing. We did not gather clinical data on patient outcomes as the recommendations from the AMS ward rounds were only theoretical, and not implemented. Finally, our calculations of staff resourcing are likely to be an underestimate as these did not include the times required to document (in the patient's 
health record) and discuss recommendations with clinical teams.

\section{Conclusion}

This pilot study has demonstrated that an AMS ward round using MedChart as the source of antimicrobial prescriptions to review is feasible and will have clinical value. A sustained service requires an adequate pool of technical and clinical staff, which needs dedicated resourcing. Given the potential health and economic benefits of AMS initiatives, health care facilities should evaluate their ability to perform initiatives such as AMS ward rounds. At our institution, there is clear scope for our pilot study to be expanded to include the Respiratory Service, as well as being a daily process. We anticipate that a trial implementation of an AMS ward round with real recommendations should include follow-up to gauge the uptake of recommendations and associated clinical outcomes.

\section{Clinical Relevance Statement}

Effective AMS programs require information on the quantity and quality of antimicrobial prescribing. In this paper, we have harnessed existing hospital information technology for comprehensive identification of candidate antimicrobial prescriptions for review in an AMS ward round. Further, we have identified the potential clinical impact of this approach with approximately $60 \%$ of reviewed prescriptions needing change to optimize antimicrobial use.

\section{Protection of Human and Animal Subjects}

In accordance with guidance from the New Zealand Health and Disability Ethics Committee (https://ethics. health.govt.nz/), this project did not require full ethical review as it was a noninterventional audit-related activity to determine the resourcing requirements for a quality improvement initiative.

\section{Conflict of Interest}

None declared.

\section{References}

1 Chan M. World Health Organization. WHO Director-General briefs UN on antimicrobial resistance. April 18, 2016. Available at: https://www.who.int/dg/speeches/2016/antimicrobial-resistance-un/en/. Accessed September 28, 2019
2 Goossens H, Ferech M, Vander Stichele R, Elseviers M for the ESAC Project Group. Outpatient antibiotic use in Europe and association with resistance: a cross-national database study. Lancet 2005;365(9459):579-587

3 World Health Organization. Global action plan on antimicrobial resistance. Geneva, Switzerland: World Health Organization Press; 2015. Available at: https://www.who.int/antimicrobialresistance/publications/global-action-plan/en/. Accessed September 28, 2019

4 Ministry of Health and Ministry for Primary Industries. New Zealand antimicrobial resistance action plan. Wellington, New Zealand: Ministry of Health; 2017. Available at: https://www.health.govt.nz/ system/files/documents/publications/new-zealand-antimicrobialresistance-action-plan.pdf. Accessed September 28, 2019

5 Interagency Task Force for Combating Antibiotic-Resistant Bacteria National action plan for combating antibiotic-resistant bacteria. Washington, USA: US Department of Health and Human Services, Centers for Disease Control and Prevention; 2015. Available at: https://www.cdc.gov/drugresistance/pdf/national_ action_plan_for_combating_antibotic-resistant_bacteria.pdf. Accessed September 28, 2019

6 Barlam TF, Cosgrove SE, Abbo LM, et al. Implementing an antibiotic stewardship program: guidelines by the Infectious Diseases Society of America and the Society for Healthcare Epidemiology of America. Clin Infect Dis 2016;62(10):e51-e77

7 Davey P, Marwick CA, Scott CL, et al. Interventions to improve antibiotic prescribing practices for hospital inpatients. Cochrane Database Syst Rev 2017;2:CD003543

8 Pharmaceutical Management Agency. Pharmaceutical Schedule (Section H). Wellington, New Zealand: Pharmaceutical Management Agency; 2019. Available at: https://www.pharmac.govt.nz/ tools-resources/pharmaceutical-schedule/section-h/. Accessed September 28, 2019

9 Dellit TH, Owens RC, McGowan JE Jr, et al. Infectious Diseases Society of America and the Society for Healthcare Epidemiology of America guidelines for developing an institutional program to enhance antimicrobial stewardship. Clin Infect Dis 2007;44(02): 159-177

10 Public Health England Start smart-then focus. Antimicrobial stewardship toolkit for English hospitals. London, England: Public Health England; 2015. Available at: https://assets.publishing.service.gov.uk/government/uploads/system/uploads/attachment_data/file/417032/Start_Smart_Then_Focus_FINAL.PDF. Accessed October 15, 2019

11 Charani E, Castro-Sanchez E, Sevdalis N, et al. Understanding the determinants of antimicrobial prescribing within hospitals: the role of "prescribing etiquette". Clin Infect Dis 2013;57(02): 188-196

12 Schuts EC, Hulscher MEJL, Mouton JW, et al. Current evidence on hospital antimicrobial stewardship objectives: a systematic review and meta-analysis. Lancet Infect Dis 2016;16(07):847-856

13 Heard KL, Hughes S, Mughal N, Azadian BS, Moore LSP. Evaluating the impact of the ICNET $®$ clinical decision support system for antimicrobial stewardship. Antimicrob Resist Infect Control 2019;8:51 